Errata

\title{
Distribution of Wounded Nucleons and of Colored Strings in $\alpha-\alpha$ Collisions at High Energies
}

\author{
A. Białas and A. Kolawa \\ Z. Phys. C - Particles and Fields 22, 231 (1984)
}

Following corrections should be made:

Table 1 and Table 2 should be interchanged. In Figs. 3-4 dotted and crossed lines should be interchanged. Furthermore, by an unfortunate omission, it was not explicitly stated that our analysis of the data of [7] was carried out following as closely as possible that of [7]. In particular, we followed the method of convoluting amplitudes which was first introduced in [7]. The conclusions of our papers are different, however, because we investigated the dependence of the theoretical predictions on model parameters which was not done in [7]. We thank Hans Bøggild for calling our attention to these mistakes.

\section{High Order Moments of the $\bar{p} p$ Multiplicity Distribution in the Dual Parton Model}

A. Capella and J. Tran Thanh Van

Z. Phys. C - Particles and Fields 23, 165 (1984)

Columns 4 and 5 of Table 2 should read:

\begin{tabular}{lcc}
\hline & $\sqrt{s}=540 \mathrm{GeV}$ & $|y|<3.5$ \\
& $K=1.4$ & $K=2$ \\
\hline$\langle N\rangle$ & 21.89 & 21.89 \\
$C_{2}$ & 1.32 & 1.35 \\
$C_{3}$ & 2.25 & 2.35 \\
$C_{4}$ & 4.77 & 5.14 \\
$D_{2} /\langle N\rangle$ & 0.56 & 0.59 \\
$D_{3} /\langle N\rangle$ & 0.67 & 0.68 \\
$D_{4} /\langle N\rangle$ & 0.91 & 0.94 \\
$\gamma_{2}$ & 0.32 & 0.35 \\
$\gamma_{3}$ & 0.29 & 0.32 \\
$\gamma_{4}$ & 0.39 & 0.43 \\
\hline
\end{tabular}

Equation (A.2) should read

$X_{k}=\frac{1}{2}\left[(1+\lambda)^{k}+(1-\lambda)^{k}\right]$. 